Historic, Archive Document

Do not assume content reflects current scientific knowledge, policies, or practices. 

BEG to call your special attention to the following list representing the different varieties of seeds that I am preparing to grow this coming season under the usual contract system, viz: subject to pro rata delivery in event of a short crop. II give my personal care and attention to the detail work of seed growing in all its branches, and claim with confidence that my stocks can not be excelled in purity, quality or any other respect, by any stocks obtainable from any other source.

\section{SPECIAL CONTRACT OFFER ORDER SHEET - CROP OF 1910 \\ For the WhOLESALE TRADE ONLY}

BAGj-Extra at market value.

SHIPMENT-As soon after harvest as practicable.

DELIVERY -F. O. B. Gilroy or Hollister.

TERMS-Net 90 days from date of shipment or 2 per cent off for cash 30 days after date of invoice.
SEED FARMS AT

GILROY
FAIR VIEW JN JUAN
STOCKTON FELIPE

DISCLAIMER-While I exercise great care to have all seeds pure and reliable, I do not give any warranty, expressed or implied, as to Description, Quality, Productiveness, or any other matter of any seeds I send out. aud I will not in any way be responsible for the crop.

\section{SPECIAL QUOTATIONS GIVEN ON APPLICATION ON 1000-POUND LOTS.}

\section{Onion}

Price, Per Ib.

\begin{tabular}{|c|c|}
\hline & Australiar Brown............. \$.60 \\
\hline & Australian Yellow Globe........ \\
\hline & Extra Early Red Flat...... \\
\hline & Large Red Wethersfield.... \\
\hline & Ohio Yellow Globe........... \\
\hline & Prize-Taker...$\ldots \ldots \ldots \ldots$ \\
\hline & Southport Yellow Globe......... \\
\hline • & Southport Red Globe.......... \\
\hline 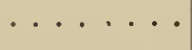 & Southport White Globe..... \\
\hline & White Portugal.......... \\
\hline & Fellow Flat Danver........... .60 \\
\hline & Yellow Globe Danver............ \\
\hline & Yellow Dutch or Strasburg...... \\
\hline .... & Philadelphia Silver Skin....... 1.20 \\
\hline
\end{tabular}

\section{Lettuce}

All Year Round............. . .25

Big Boston................... .25

Boston Curled.................. .25

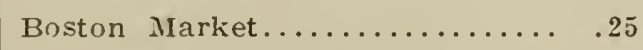

Black-Seeded Simpson......... .25

Brown Dutch................. .25

Black-Seeded Tennisball........ .25

Black-Seeded Butter........... .25

Crisp As Ice.................. .25

California Cream Butter....... .25

\section{Lettuce}

\begin{tabular}{|c|c|c|}
\hline 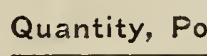 & Price, $F$ & Per \\
\hline 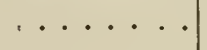 & Deacon .............. & \\
\hline & Drumhead or Malta...... & $\$ .25$ \\
\hline & Denver Market.......... & .25 \\
\hline & Early Curled Silesia... & .25 \\
\hline & Grand Rapids.......... & .25 \\
\hline & Golden Queen................ & .25 \\
\hline & Henderson's New Iork Improved & .25 \\
\hline & Hubbard Market........... & .25 \\
\hline & rg $\ldots \ldots \ldots \ldots$ & .25 \\
\hline . & Immensity $\ldots \ldots \ldots \ldots \ldots$ & .25 \\
\hline$\cdots$ & Improved Hanson......... & .25 \\
\hline & Mignonette...$\ldots \ldots \ldots \ldots \ldots$ & .25 \\
\hline & Mammoth Black-Seeded Butter... & .25 \\
\hline & May King................ & .25 \\
\hline & ssion $\ldots \ldots \ldots \ldots$ & .25 \\
\hline & hiladelphia Butter... & .25 \\
\hline & Prizehead .. & .25 \\
\hline & Salamander ..... & .25 \\
\hline & St. Louis Butter...... & .25 \\
\hline & Tombannock ........... & .25 \\
\hline & White-Seeded Tennisball.... & .25 \\
\hline & Paris White Cos........... & .25 \\
\hline & Trianon Cos........... & .25 \\
\hline & Yellow-Seeded Butter... & .25 \\
\hline & White-Seeded Simpson..... & .25 \\
\hline & Vhite Summer Cabbage... & \\
\hline
\end{tabular}




\section{Radish}

Quantity, Pounds

Price, Per Ib.
Vicks Scarlet Globe.........\$ .15

Scarlet Turnip, white tip....... .15

crimson Giant ..................

Early Scarlet Turnip.............. .15

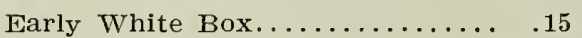

French Breakfast............. .15

Long Scarlet, short top........ .15

Long Brightest Scarlet......... .15

Icicle ................... .15

Chartier .....................15

Long White Vienna............ .15

White Strasburg.............. .15

China Rose Winter........... .15

Long Black Spanish........... .15

\section{Salsify}

Mammeth Sandwich Island.....\$ .35

\section{Sweet Peas}

Varietics marked $(*)$ are best of their color.

THITE.

Mount Blane................. . 12

Emily Henderson.............. .12

Blanche Burpee.................. .12

Burpee's Earliẹst White........ .25

*Burpee's White Spencer ....... .40

*Dorothy Eckford.............. .12

* Nora Unwir................. .20

Sadie Burpee Black Seed........ .12

Sadie Burpee White Seed....... .12

"Shasta .................. .12

White Wonder.................. .12

CREAM OR LIGHT YELLOW.

*Burpee's Primrose Spencer..... . .40

Beacon ................... .12

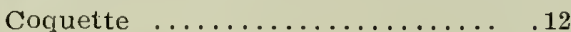

*Dora Breadmore............. .12

Earliest Sunbeams..............25

"Hon. Mrs. E. Kenyon......... .12

*Mrs. Collier.................. .12

James Grieve .............. .12

Queen Victoria................ .12

"Sybil Eckford................ .12

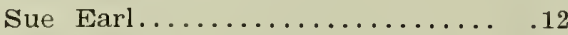

*Stella Morse.................12

BLUSH PINK.

Modesty

\section{Sweet Peas}

Quantity, Pounds

Price, Per lb.

Agnes Eckford................. .12

Countess Lathom............ .12

Crotvn Jewel................12

Florence Spencer............. .40

Jeanie Gordon............... .12

*Katherine Tracy.............. .12

"Lovely .................... .12

*Mrs. Alfred Watkins.......... .20

*Countess Spencer (true)...... .40

*Gladys Unwin.............. .20

SHADES OF PINK.

Apple Blossom...............12

Bolton Pink.................. .12

"Dainty .................... .12

*Enchantress .............. .50

Janet Scott................... .12

Marchioness of Cholmondelay.... $\quad .12$

*Paradise ................ .50

"Prima Donna............... .12

Royal Rose............... .12

Queen of Spain............... .12

SHADES OF ROSE.

E. J. Castle..................20

Lord Rosebery............... .12

*John Ingham.............. .12

"John Ingham, waved......... .40

George Herbert............. .25

Mrs. Dugdale................ .12

*Phyllis Unwin............. .20

*Prince of Wales............. .12

BRIGHT RED AND SCARLET.

Coccinea ................... .12

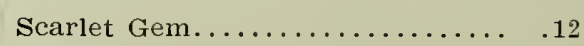

Fircfly $\ldots \ldots \ldots \ldots \ldots \ldots \ldots \ldots . .12$

Mars ...................... .12

Prince Edward of York........ .12

*Queen Alexandra............ .12

Salopian (Improved Sunproof)... $\quad .12$

"King Edward VII........... .12

SHADES OF ORANGE.

*Evelyn Byatt.............. .12

Gorgeous ................ .12

*Helen Lewis................ .25

*Henry Eckford.............. .12

Hon. F. Bouverie............ .12

....... Lady Marie Currie.............. . .

..... . Miss Wilmott.............. .12

$\ldots .$. Venus ................... .12 


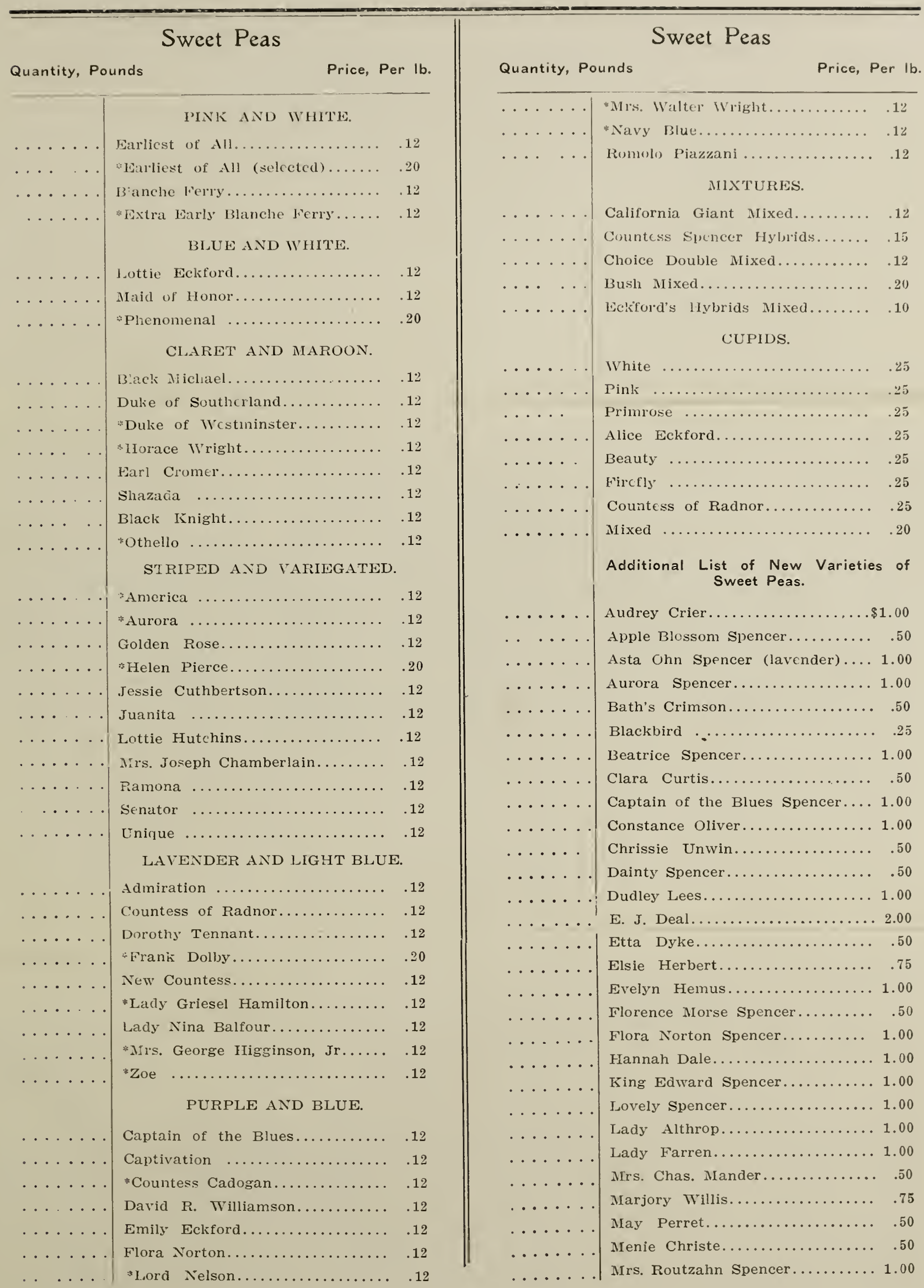




\section{Sweet Peas}

Quantity, Pounds

Price, Per lb.

\begin{tabular}{|c|c|}
\hline$\cdots \cdots \cdots$ & Mrs. Sankey Spencer.......... 1.00 \\
\hline$\cdot \cdot \cdot$ & Mrs. Joseph Chamberlain Spencer .50 \\
\hline$\ldots$ & Mrs. Henry Bell....... \\
\hline & Mrs. Bierberstedt............ .50 \\
\hline & Mrs. R. W. Pitt........... \\
\hline & Mina Johnson.. \\
\hline & Mrs. A. Ireland. \\
\hline & Marbled Blue................ \\
\hline & Mrs. Walter Wright Spencer... 1.00 \\
\hline & Nell Gwynne......... \\
\hline & Olive Ruffel....... \\
\hline & Othello Spencer....... \\
\hline & Prince Olaf........... \\
\hline & Prince of Asturias............ \\
\hline$\cdot$. & Princess Victoria.............. \\
\hline$\cdots$ & Purity $\ldots \ldots \ldots \ldots \ldots \ldots \ldots$ \\
\hline$\cdot \cdot \cdot$ & Princess Alice Spencer......... \\
\hline & Princess Katherine Spencer..... .5 \\
\hline & Paradise Red Flake (Dark Stripe) 1.00 \\
\hline & Paradise Red Flake (Light \\
\hline & Stripe) $\ldots \ldots \ldots \ldots$ \\
\hline & Paradise Ivory.... \\
\hline & Pink Pearl..... \\
\hline & Rosie Adams ......... \\
\hline & Ramona Spencer...... \\
\hline & Senator Spencer....... \\
\hline & St. Georgc....... \\
\hline & Starks Elegance... \\
\hline & Sutton's Queen......... \\
\hline & The Marquis........... \\
\hline & Tennant Spencer. \\
\hline & he \\
\hline
\end{tabular}

Asters

Quantity, Pounds Price, Per lb.

\begin{tabular}{|c|c|}
\hline$\cdots$ & 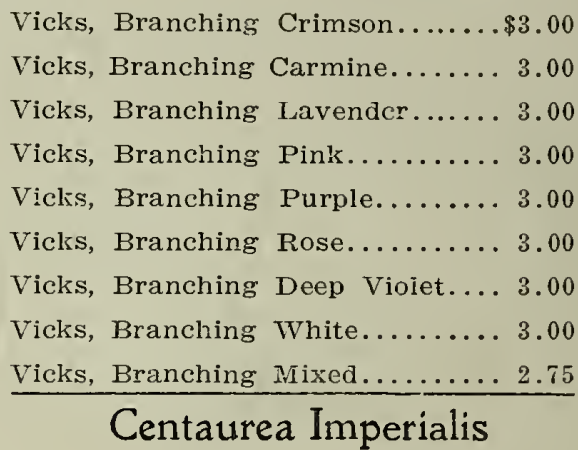 \\
\hline & 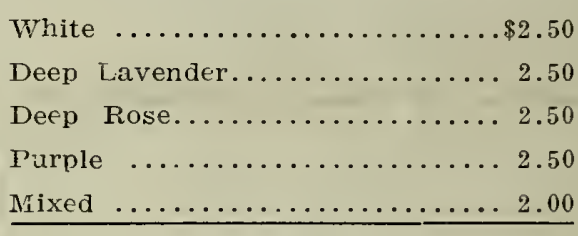 \\
\hline & Cosmos \\
\hline & 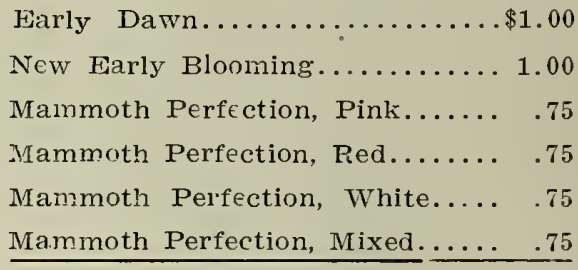 \\
\hline & Verbena \\
\hline$\cdots \cdots$ & $\begin{array}{l}\text { Mammoth Auricula-flowered.... } \$ 4.00 \\
\text { Mammoth Italian, Striped..... } 4.00 \\
\text { Mammoth, Purple........... } 4.00 \\
\text { Mammoth, Pink............ } 4.00 \\
\text { Mammoth, White.......... } 4.00 \\
\text { Mammoth, Fordhook, Mixed.... } 4.00 \\
\text { Scarlet Defiance............. } 4.00\end{array}$ \\
\hline
\end{tabular}

\section{WALDO ROHNERT, GILROY, CAL:}

Dear Sir-Please enter our order for the above named quantities of seeds at prices annexed upon the terms and conditions herein specified.

\section{Yours truly,}

